\title{
Therapeutic potential of a dual mTORC1/2 inhibitor for the prevention of posterior capsule opacification: An in vitro study
}

\author{
HAO FENG $^{1}$, ZHIBO YANG $^{2}$, XUE BAI $^{1}$, MEIRONG YANG $^{1}$, YUAN FANG $^{1}$, \\ XIAONAN ZHANG ${ }^{1}$, QIQIANG GUO ${ }^{3}$ and HONG NING ${ }^{1}$
}

\author{
${ }^{1}$ Department of Ophthalmology, The First Hospital of China Medical University; ${ }^{2}$ Department of Ophthalmology, \\ The Fourth People's Hospital of Shenyang; ${ }^{3}$ Key Laboratory of Medical Cell Biology, College of Translational Medicine, \\ China Medical University, Shenyang, Liaoning 110001, P.R. China
}

Received July 14, 2017; Accepted January 10, 2018

DOI: $10.3892 /$ ijmm.2018.3398

\begin{abstract}
Mammalian target of rapamycin (mTOR) serves a central role in regulating cell growth and survival, and has been demonstrated to be involved in the pathological progression of posterior capsule opacification (PCO). In the present study, the potency of PP242, a novel dual inhibitor of mTOR complex $1 / 2$ (mTORC1/2), in the suppression of the growth of human lens epithelial cells (HLECs) was investigated. Using a Cell Counting Kit- 8 and a wound healing assay, it was demonstrated that PP242 inhibited the proliferation and migration of HLECs. In addition, western blot analysis indicated that PP242 completely inhibited mTORC1 and mTORC2 downstream signaling activities, whereas rapamycin only partially inhibited mTORC1 activity within LECs. Furthermore, PP242 treatment led to an upregulation of the expression levels of p53 and B cell lymphoma-2 (Bcl-2)-associated X and downregulation of $\mathrm{Bcl}-2$. In addition, flow cytometric analysis demonstrated that PP242 induced the cell cycle arrest at the G0/G1 phase, which may have caused apoptosis and induced autophagy within the LECs. The results of the present study suggested that administration of PP242 may potentially offer a novel therapeutic approach for the prevention of PCO.
\end{abstract}

\section{Introduction}

Modern surgical techniques for cataracts are continuously improving; however, posterior capsule opacification (PCO) remains common long-term complication that results in decreased visual acuity following successful surgery $(1,2)$.

Correspondence to: Professor Hong Ning, Department of Ophthalmology, The First Hospital of China Medical University, 155 Nanjing North Street, Heping, Shenyang, Liaoning 110001, P.R. China

E-mail: leary1000@163.com

Key words: posterior capsule opacification, mammalian target of rapamycin complex 1/2, PP242, protein kinase B, lens epithelial cells
Without posterior capsulorhexis, almost every child or infant suffers a visual decline caused by PCO following cataract surgery, while the incidence rate of PCO in adults is $\geq 50 \%$ at 2-5 years post-surgery $(3,4)$. Artificial intraocular lenses (IOLs) have been developed for the prevention of PCO and have well-designed sharp borders at the optic edge or on the capsular tension rings, which may delay cell migration into the posterior capsule $(5,6)$. However, patients who have been implanted with these lenses still undergo neodymium yttrium aluminum garnet laser capsulotomy, the only effective treatment for PCO, albeit at a lower rate (7). This procedure is associated with rhegmatogenous retinal detachment, cystoid macular edema and damage to the intraocular lens (8), and is not suitable for infants following congenital cataract surgery (3).

PCO has a complex pathogenesis and is induced by cataract surgery as an inflammatory and wound-healing response (2), which includes lens epithelial cell (LEC) attachment, proliferation, spreading and migration toward the capsular bag surface of the IOL. Damaged ocular tissue releases chemokines, which attract cells of the immune system to remove damaged tissue and to facilitate subsequent tissue repair (9). Therefore, post-surgical pharmacological inhibition of LEC proliferation and migration, and the promotion of LEC apoptosis are possible options for preventing PCO.

In the process of $\mathrm{PCO}$ development, the phosphoinositide 3-kinase (PI3K)/AKT/mammalian target of rapamycin (mTOR) signaling pathway serves a crucial role. mTOR is a serine/threonine kinase at the nexus between oncogenic PI3K/AKT signaling and critical downstream pathways that drive cell growth, survival and resistance to therapeutic agents (10-12). Previously, numerous studies have revealed that the activation of the AKT/mTOR signaling pathway is a key regulator in the proliferation and migration of LECs following stimulation by certain growth factors, including hepatocyte growth factor (HGF), platelet-derived growth factor, insulin-like growth factor (IGF), fibroblast growth factor (FGF) and integrin-linked kinase (13-16).

PP242, as a new-generation mTOR inhibitor, is able to completely suppress mTOR complex 1 (mTORC1) as well as mTORC2-mediated signaling pathways by suppressing the AKT feedback loop (17). Most importantly, PP242 has been 
reported to markedly improve antitumor activity in vivo and in vitro, and the effectiveness of this drug in cancer treatment has been assessed in clinical trials (18). Being more effective than rapamycin, PP242 may be an attractive candidate for the post-operative prevention and treatment of PCO following cataract surgery.

The present study was performed to investigate the capacity of PP242 to inhibit the crucial cellular events in the formation of PCO, involving LEC proliferation, attachment and migration, and the induction of LEC autophagy, apoptosis and/or death compared with that of rapamycin. The present results may contribute to the development of a novel strategy to prevent $\mathrm{PCO}$.

\section{Materials and methods}

Cell lines and reagents. The SRA01/04 cell line constituting human LECs (HLECs) was purchased from The Cell Bank of the Chinese Academy of Sciences (Shanghai, China). SRA01/04 cells were maintained in Dulbecco's modified Eagle's medium (GE Healthcare Life Sciences, Little Chalfont, UK) with $10 \%$ fetal bovine serum (FBS; GE Healthcare Life Sciences), penicillin (100 U/ml) and streptomycin $(100 \mu \mathrm{g} / \mathrm{ml})$. All cells were maintained at $37^{\circ} \mathrm{C}$ in a humidified incubator with $95 \%$ air and $5 \% \mathrm{CO}_{2}$.

PP242 was purchased from Selleck Chemicals (Houston, TX, USA). The rabbit monoclonal anti-mTOR (cat. no. 2983; 1:1,000), rabbit monoclonal anti-phosphorylated (p)-mTOR (Ser2448; cat. no. 5536; 1:1,000), rabbit monoclonal anti-p-p70S6K (Thr389; cat. no. 9234; 1:1,000), rabbit polyclonal anti-p-p70S6K (Ser371; cat. no. 9208; 1:1,000), rabbit polyclonal anti-p70S6K (cat. no. 9202; 1:1,000), rabbit monoclonal anti-p-4E-BP1 (Thr37/46; cat. no. 2855; 1:1,000), rabbit monoclonal anti-p-AKT (Ser473; cat. no. 4058, 1:1,000), rabbit monoclonal anti-AKT (cat. no. 4685, 1:1,000), rabbit monoclonal anti-microtubule-associated protein light chain 3 (LC3) A/B (cat. no. 12741; 1:1,000), rabbit polyclonal anti-cleaved caspase-3 (cat. no. 9661; 1:1,000), rabbit polyclonal anti-cleaved poly(ADP ribose) polymerase (PARP; cat. no. 9541; 1:1,000), and mouse monoclonal anti-B-cell lymphoma 2 (Bcl-2; cat. no. 15071; 1:1,000) were purchased from Cell Signaling Technology, Inc. (Danvers, MA, USA). Rabbit monoclonal anti-Bcl-2-associated X protein (Bax; cat. no. ab182733 1:1,000) was purchased from Abcam (Cambridge, UK). Mouse monoclonal anti-p53 (cat. no. sc-47698; 1:1,000) and mouse monoclonal anti-cyclin D1 (cat. no. sc-70899; 1:1,000) were purchased from Santa Cruz Biotechnology, Inc. (Dallas, TX, USA). Mouse monoclonal anti- $\alpha$-tubulin (cat. no. AC012; 1:5,000), horseradish peroxidase (HRP) goat anti-rabbit immunoglobulin $\mathrm{G}(\mathrm{IgG} ; \mathrm{H}+\mathrm{L}$; cat. no. AS014; dilution, 1:5,000), HRP goat anti-mouse $\mathrm{IgG}(\mathrm{H}+\mathrm{L}$; cat no. AS003; $1: 5,000)$ were purchased from ABclonal Biotech, Co., Ltd. (Wuhan, China).

Cell proliferation assays. A Cell Counting Kit-8 assay (CCK-8; Nanjing KeyGen Biotech Co., Nanjing, China) was used to assess the effects of PP242 on SRA01/04 cell growth according to the manufacturer's protocols. Cells were incubated at $37^{\circ} \mathrm{C}$ with $0,100,250,500,750$ or $1,000 \mathrm{nM}$ PP2 42 for $24 \mathrm{~h}$. The absorbance was measured at a wavelength of $450 \mathrm{~nm}$ using a plate reader (model 680; Bio-Rad Laboratories, Inc., Hercules, CA, USA).

Colony formation assay. SRA01/04 cells were counted and seeded into $3.5-\mathrm{cm}$ dishes at a density of 1,000 cells per dish in regular culture medium containing various doses of rapamycin $(0.5$ or $1.0 \mu \mathrm{M})$ or PP242 $(0.5,1.0$ or $2.0 \mu \mathrm{M})$. The cells were cultured for 10 days, during which the medium was refreshed every 3 days, until visible clones appeared. The cells were washed with PBS, fixed with $4 \%$ paraformaldehyde for 15 min at room temperature and stained with Giemsa's solution for $20 \mathrm{~min}$ at room temperature. Images of colonies in each dish were captured using a digital camera.

Wound healing migration assay. Cells were incubated in the presence or absence of PP242 $(1,000 \mathrm{nM})$ at $37^{\circ} \mathrm{C}$ for 24 or 36 h. Following serum starvation of confluent SRA01/04 cells for $2 \mathrm{~h}$ in 6-well plates, the media was replaced with DMEM without supplementation; line-shaped wounds were generated across the cell monolayers using a sterile pipette tip. At 0,24 and $36 \mathrm{~h}$, images of the wounded cell layers were captured under bright field using an Olympus IX-71 inverted fluorescence microscope (Olympus Corp., Tokyo, Japan). ImageJ v1.51 software (National Institutes of Health, Bethesda, MD, USA) was used to quantify the closure of the wound gap area, which represented the migratory potential of the cells.

Transwell assay. SRA01/04 cells $\left(3 \times 10^{4}\right)$ were suspended in $100 \mu \mathrm{l}$ serum-free medium and then applied to the upper inserts of a Transwell plate (with 8.0- $\mu$ m-pore polycarbonate membranes; Corning Life Science, Corning, NY, USA) and $600 \mu \mathrm{l}$ DMEM with $10 \%$ FBS was added to the lower chambers. Following incubation with rapamycin $(1.0 \mu \mathrm{M})$ or PP242 $(0.5,1.0$ or $2.0 \mu \mathrm{M})$ at $37^{\circ} \mathrm{C}$ for $12 \mathrm{~h}$, cells in the upper inserts were removed with cotton swabs, while the cells on the lower side of the membrane were then fixed with methanol for $10 \mathrm{~min}$ at room temperature, stained with hematoxylin for $15 \mathrm{~min}$ at room temperature and counted under a Nikon Eclipse Ni-U microscope (Nikon Corp., Tokyo, Japan).

Immunocytochemistry assay. SRA01/04 cells were seeded into 24-well plates at $1 \times 10^{4}$ cells/well and cultured on glass coverslips overnight. After serum starvation for $2 \mathrm{~h}$, the cells were treated with 0,250 or $500 \mathrm{nM}$ PP242 for $24 \mathrm{~h}$. Following treatment, the cells were rinsed once with PBS, fixed in fresh $4 \%$ paraformaldehyde for $15 \mathrm{~min}$ at room temperature and permeabilized with $0.1 \%$ Triton X-100. The cells were blocked with $5 \%$ bovine serum albumin (Gen-View Scientific Inc., El Monte CA, USA) in PBS for $1 \mathrm{~h}$ at room temperature and incubated overnight with anti-p-AKT (Ser473) antibody (dilution, 1:200) at $4^{\circ} \mathrm{C}$, followed by incubation with Alexa Fluor $^{\circledR} 488$ goat anti-rabbit IgG $(\mathrm{H}+\mathrm{L})$ (1:500; A-11034, dilution, Thermo Fisher Scientific, Inc., Waltham, MA, USA) for $1 \mathrm{~h}$ at room temperature. The p-AKT (Ser473) protein was detected using fluorescence microscopy at 450-490 nm.

Western blot analysis. To determine the expression profile of proteins, whole-cell extracts were prepared from $1 \times 10^{6}$ cells in radioimmunoprecipitation assay lysis buffer [50 mM Tris/HCl, pH 7.4, 150 mM NaCl, 1\% Nonidet P-40, 
$0.25 \%$ Na-deoxycholate, $1 \mathrm{mM}$ EDTA and protease inhibitor cocktail (B14001, Bimake, Houston, TX, USA)]. Protein concentrations were determined via a Bradford protein assay. Aliquots of samples containing $30 \mu \mathrm{g}$ denatured protein were loaded on 10 or $15 \%$ polyacrylamide gels respectively. Samples were separated by SDS-PAGE and transferred to polyvinylidene fluoride (PVDF) membranes (Merck KGaA, Darmstadt, Germany). The membranes were blocked with 5\% nonfat dry milk in $20 \mathrm{mM}$ Tris (pH 7.4), $137 \mathrm{mM} \mathrm{NaCl}$ and $0.05 \%$ Tween-20 for $1 \mathrm{~h}$ at room temperature. The membranes were probed with the primary antibodies overnight at $4{ }^{\circ} \mathrm{C}$ in Tris-buffered saline with Tween-20. The expression of mTOR, p-mTOR, p-AKT (Ser473), AKT, p-p70S6K (Thr389), p-p70S6K (Ser371), p70S6K, p-4EBP1, cyclin D1, p53, Bax, Bcl-2, cleaved caspase-3, cleaved-PARP, LC3 A/B, was detected using the same specific primary antibodies as aforementioned and tubulin was used as the loading control. All of the PVDF membranes were analyzed by chemiluminescence (Tanon, Tanon Science \& Technology Co., Ltd., Shanghai, China).

Cell-cycle analysis by flow cytometry. SRA01/04 cells were seeded into 6 -well plates at $3 \times 10^{5}$ cells/well and incubated with different concentrations of PP242 (0, 250, 500, 750 and $1,000 \mathrm{nM}$ ) for $48 \mathrm{~h}$ at $37^{\circ} \mathrm{C}$. The cells were then collected, washed with PBS and suspended in a staining buffer $[10 \mu \mathrm{g} / \mathrm{ml}$ propidium iodide, $0.5 \%$ Tween 20 and $0.1 \%$ RNase A (ST578; Beyotime Institute of Biotechnology, Shanghai, China) in PBS]. The cells were analyzed using a BD FACSCalibur flow cytometer (BD Biosciences, San Jose, CA, USA) with the analysis software program ModFit LT v3.0 (Verity Software House, Inc., Topsham, ME, USA). The gating was set to exclude cell debris, doublets and clumps.

Cell apoptosis analysis by flow cytometry. SRA01/04 cells were seeded into 6 -well plates at $3 \times 10^{5}$ cells/well and treated with PP242 $(0,0.5,1,1.5$ and $2 \mu \mathrm{M})$ for $48 \mathrm{~h}$ in serum-free medium, washed with ice-cold PBS and double-stained with annexin-V-allophycocyanin (APC) and 7-aminoactinomycin D (7-AAD) to quantify the percentage of apoptotic cells using a BD LSRFortessa flow cytometer (BD Biosciences). Cells incubated in the binding buffer with only annexin-V-APC or 7-AAD served as controls. The results were analyzed with the software program BD FACSDiva 7.0 (BD Biosciences).

Statistical analysis. Values are expressed as the mean \pm standard deviation of three independent experiments. Statistical significance was analyzed using a Student's t-test or a one-way analysis of variance followed by a Tukey's multiple comparisons test using the SPSS 17.0 (SPSS Inc., Chicago, IL, USA) and Prism 5.0 software (GraphPad Software, Inc., LA Jolla, CA, USA). $\mathrm{P}<0.05$ was considered to indicate a statistically significant difference.

\section{Results}

Targeted inhibition of mTORC1/2 signaling by the mTOR kinase inhibitor PP242 effectively suppresses LEC proliferation and migration. The effects of targeted inhibition of mTORC1/2 on LEC proliferation were examined using a CCK-8 assay. As presented in Fig. 1A, PP242-treated SRA01/04 cells proliferated more slowly compared with cells in the control group. Treatment with 100-1,000 nM PP242 for $24 \mathrm{~h}$ reduced the viability of the LEC lines in a dose-dependent manner $(\mathrm{P}<0.01)$. In addition, the results of the colony formation assay revealed that compared with rapamycin, PP242 inhibited the clone forming ability of SRA01/04 cells more effectively (Fig. 1B). From the cell survival curve, the half-maximal inhibitory concentration $\left(\mathrm{IC}_{50}\right)$ of PP242 on the SRA01/04 cell line was determined to be $2.159 \mu \mathrm{M}$. The difference in cell survival curves between rapamycin and PP242 demonstrated that the inhibitory effect of PP242 on the proliferation of SRA01/04 cells was stronger than that of rapamycin (Fig. 1B).

Subsequently, a wound healing assay and a Transwell assay were performed to confirm the inhibitory effect of PP242 on LEC migration. The cell migratory ability was evaluated at 24 and $36 \mathrm{~h}$ of exposure to 1,000 nM PP242. As presented in Fig. $1 \mathrm{C}$ and E, the larger gap area of the wound demonstrated that SRA01/04 cells treated with PP242 migrated more slowly compared with the cells in the control group $(\mathrm{P}<0.05)$. Cell chemotactic motility was evaluated at $12 \mathrm{~h}$ of exposure to rapamycin or PP242. The results of the present study revealed significant decreases in the number of cells on the bottom side of the Transwell inserts in the rapamycin and PP242 groups compared with those in the control group. However, compared with that in the rapamycin group, the number of cells which migrated to the lower side of the membrane exhibited a more significant decrease following treatment with PP242 $(\mathrm{P}<0.01$; Fig. 1D and F).

PP242, but not rapamycin, inhibits $m T O R C 1$ as well as mTORC2 function in LECs. In the present study, the effects of PP242 on the AKT/mTOR signaling pathway in LECs were detected via western blot analysis. As expected, the formation of p-AKT (Ser473) was significantly inhibited by PP242, indicating that PP242 may block mTORC2 in the LECs. Western blot analysis revealed that the protein expression levels of p-AKT (Ser473) decreased at $2 \mathrm{~h}$ following $500 \mathrm{nM}$ PP242 treatment and the efficiency of inhibition increased with increasing concentrations of PP242 (Fig. 2A-D). The phosphorylation of mTOR was also detected, which demonstrated the same tendency as p-AKT (Ser473) regarding PP242-mediated inhibition. The mTOR signaling pathway has been reported to control cell growth primarily by regulating cap-dependent translation and ribosome biogenesis via the phosphorylation of p70S6K and 4EBP1, respectively, by mTORC1 (19). Therefore, p-p70S6K (Thr389 and Ser371) and p-4EBP1 were assessed as indicators of mTORC1 activation. The results revealed that mTORC1 was almost completely inhibited by PP242 in the LECs at a concentration of $100 \mathrm{nM}$ and a short incubation time of $2 \mathrm{~h}$. An immunocytochemical staining assay indicated that the fluorescence intensity of p-AKT (Ser473) was also markedly decreased by PP242 treatment compared with that in the control group (Fig. 2E). Conversely, the first-generation mTOR inhibitor rapamycin did not reduce but increase p-AKT Ser473. In addition, rapamycin did not significantly inhibit the activation of p-mTOR and also failed to block the phosphorylation of 4EBP1 in the present study. In addition, rapamycin inhibited the phosphorylation of p70S6K; however, the results of the present study revealed that rapamycin failed to inhibit 

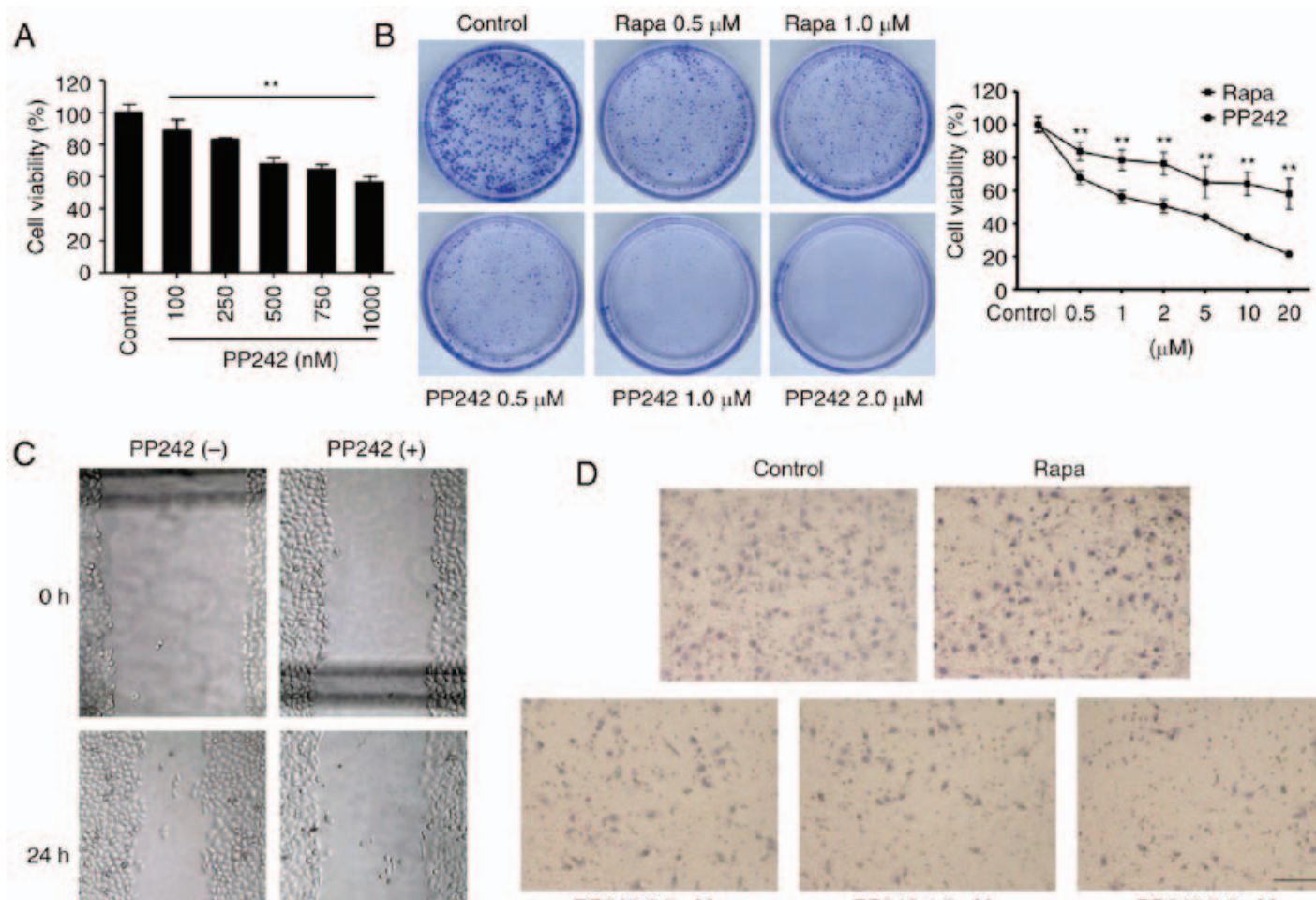

Control
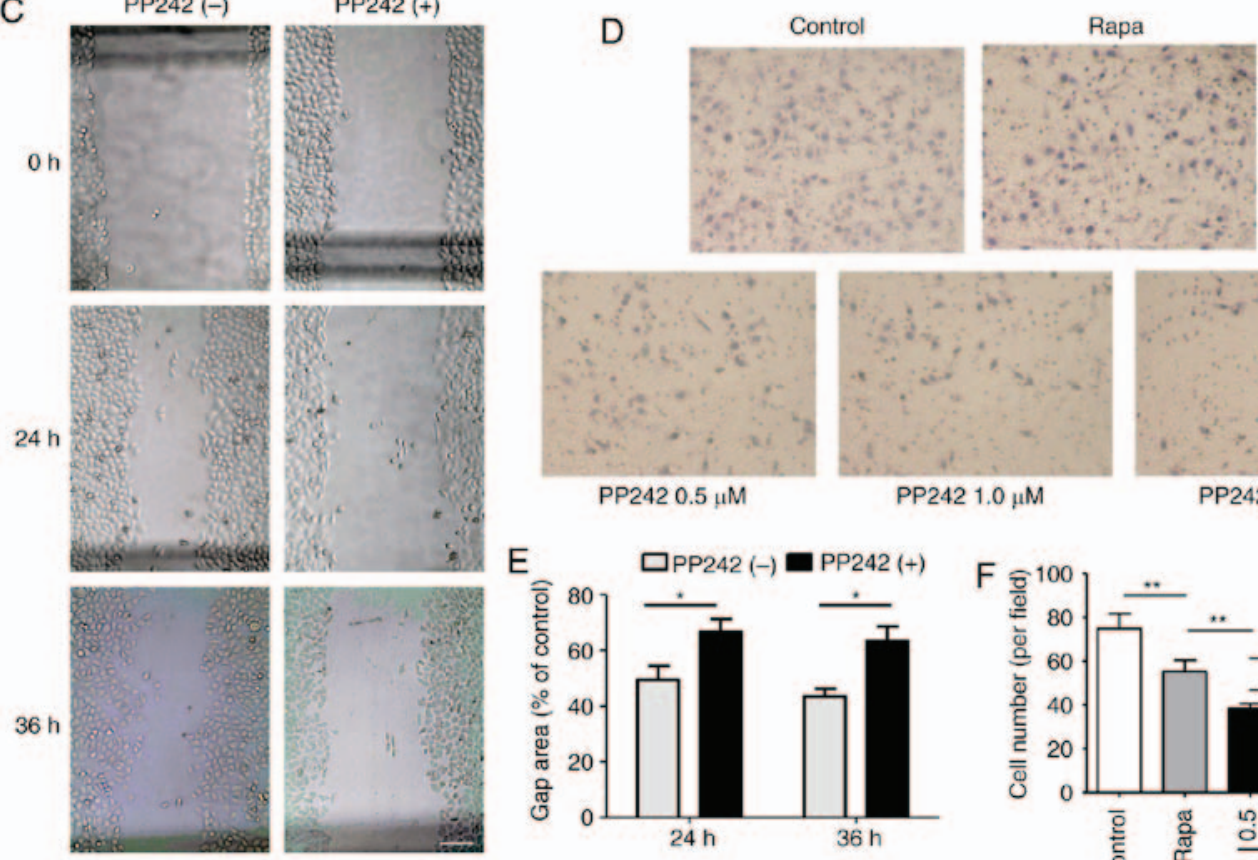

PP242 $0.5 \mu \mathrm{M}$

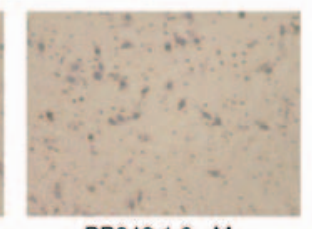

PP242 $1.0 \mu \mathrm{M}$
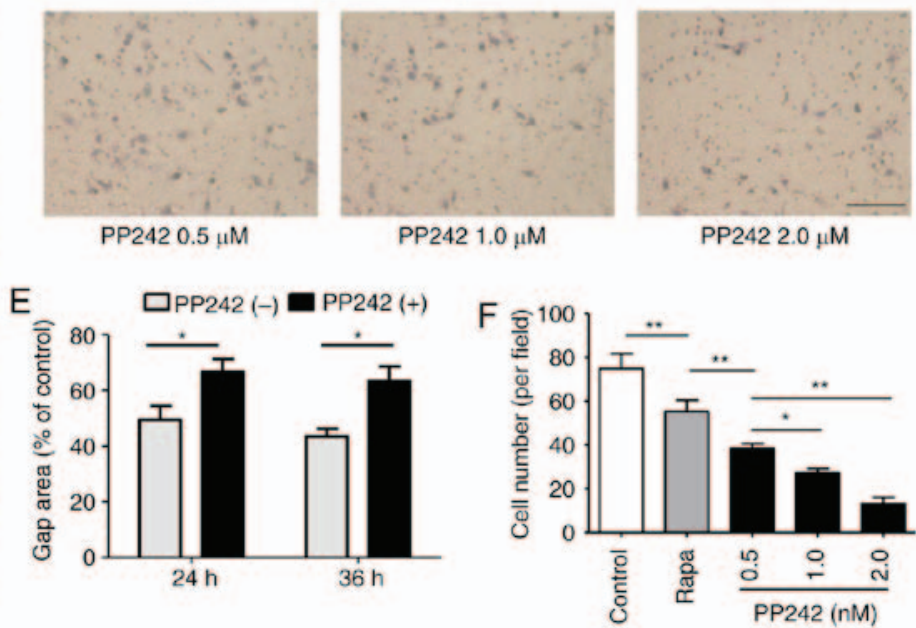

Figure 1. PP242 inhibits the proliferation and migration of a human lens epithelial cell line. (A) PP242 inhibited the survival of SRA01/04 cells in a dose-dependent manner. SRA01/04 cells were treated with various doses of PP242 (0, 100, 250, 500, 750 and 1,000 nM) for $24 \mathrm{~h}$. A Cell Counting Kit-8 assay was used to determine cell viability. (B) A plate colony formation assay (left panel; dish diameter=3.5 cm) was used to examine the proliferation of SRA01/04 cells following treatment with vehicle, Rapa $(0.5$ and $1.0 \mu \mathrm{M})$, or PP242 $(0.5,1.0$ and $2.0 \mu \mathrm{M})$. Cell survival curves (right panel) for Rapa and PP242 demonstrated that the inhibitory effect of PP242 on the proliferation of SRA01/04 cells was stronger than that of Rapa. The half-maximal inhibitory concentration of PP242 on the SRA01/04 cell line was determined to be $2.159 \mu \mathrm{M}$. (C) A wound healing assay was used to examine the migration of SRA01/04 cells following treatment with vehicle or PP242 (1,000 nM, 24 or $36 \mathrm{~h}$; scale bar=100 $\mu \mathrm{m})$. (D) A Transwell assay was performed on SRA01/04 cells treated with Rapa $(1.0 \mu \mathrm{M})$ or PP242 $(0.5,1.0$ or $2.0 \mu \mathrm{M})$. (E) Quantification of the wound gap area associated with the cell migration ability. PP242 significantly restrained the migration of SRA01/04 cells (scale bar $=100 \mu \mathrm{m}$ ). (F) Quantification of the migration ability of the cells (E). Values are expressed as the mean \pm standard deviation. ${ }^{*} \mathrm{P}<0.05$, ${ }^{* *} \mathrm{P}<0.01$ vs. control. Rapa, rapamycin.

mTOR pathway activity, but only partly inhibited the activity of mTORC1 compared with that of PP242.

PP242 induces cell cycle arrest of LECs in G0/G1-phase by downregulating cyclin D1. To investigate the effects of PP242 on the cell cycle progression of LECs, SRA01/04 cells were analyzed by flow cytometry. As presented in Fig. 3A and B, treatment with PP242 $(0,250,500,750$ and $1,000 \mathrm{nM})$ for $48 \mathrm{~h}$ induced marked cell cycle arrest in G0/G1-phase. Fluorescence-assisted cell sorting (FACS) analysis revealed that a 48-h exposure to PP242 increased the G0/G1 phase population in a dose-dependent manner $(\mathrm{P}<0.05)$. To investigate the underlying mechanisms of the inhibition of the cell cycle of LEC by PP242, the protein expression levels of cyclin D1, which serves an important role in the progression of the cell cycle, were analyzed via western blot analysis. As presented in Fig. 3C and D, PP242 significantly decreased the cyclin D1 expression levels in the LECs. These results suggested that PP242 suppressed cell proliferation and inhibited cell cycle progression by downregulating cyclin D1 expression and causing G0/G1 phase arrest.

Effect of PP242 on p53, Bax and Bcl-2 protein expression in LECs. While p53 has long been recognized as a tumor suppressor, it is indisputably important in normal cell functions as a sequence-specific transcription factor that may inhibit cell cycle progression, promote senescence or induce apoptotic cell death following activation (20). In the lens, p53 overexpression may counteract the proliferation of nontumor cells. Previous studies have reported that in lens-specific E1A expression transgenic mice, CREB-binding protein/p300 activity induced LEC apoptosis was required for activation 


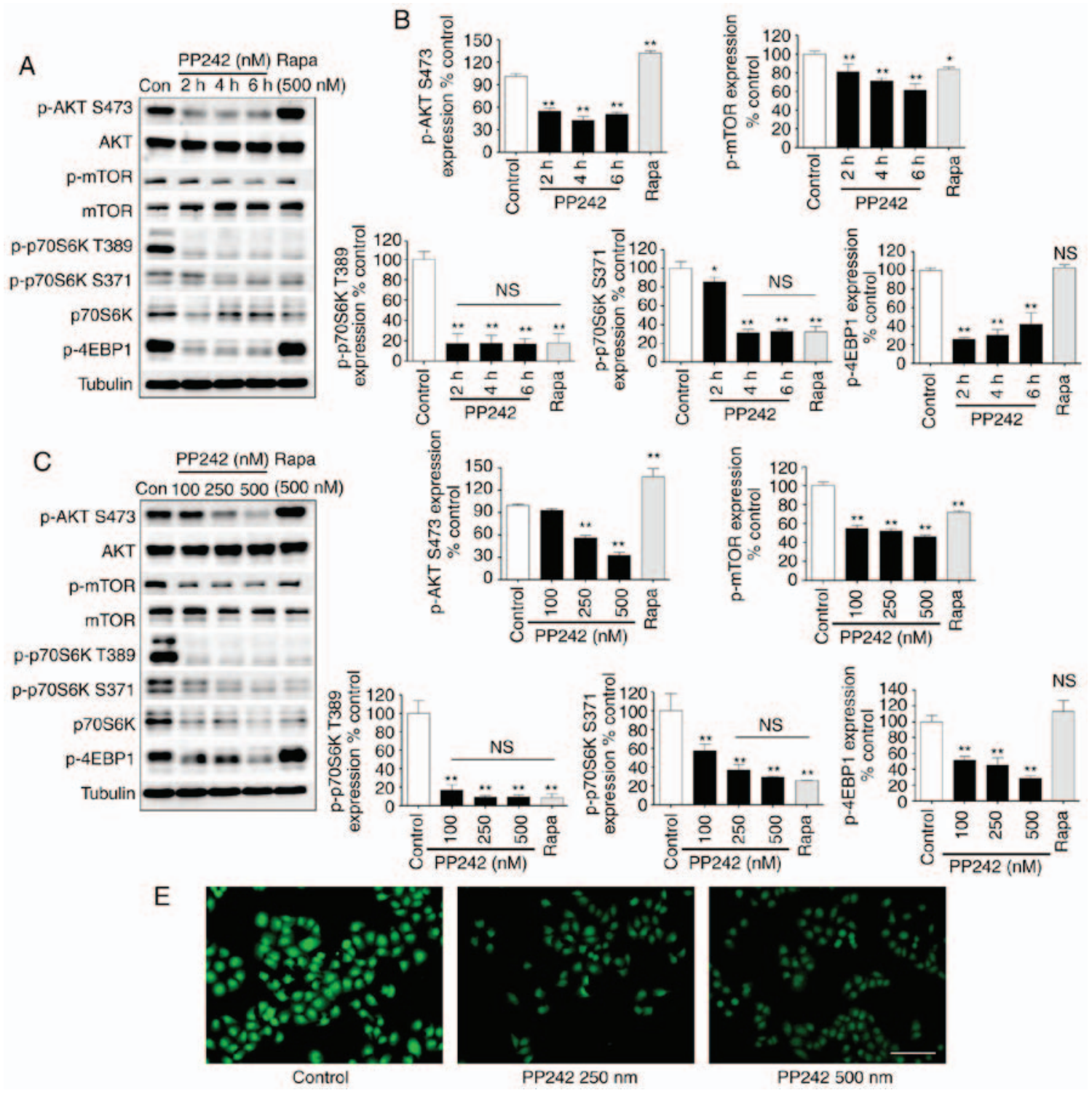

Figure 2. PP242 blocks the AKT/mTOR pathway by inhibiting mTORC1 and mTORC2 signaling in lens epithelial cells. (A) Targeted inhibition of mTORC1/2 signaling by PP242 in SRA01/04 cells exhibited a significant effect. SRA01/04 cells were incubated with $500 \mathrm{nM}$ Rapa for $6 \mathrm{~h}$ or the indicated times with $500 \mathrm{nM}$ PP242. Western blot analysis of p-AKT (Ser473), AKT, p-mTOR, mTOR, p-p70S6K (Thr389 and Ser371), p70S6K and p-4EBP1 and (B) quantitative analysis was performed. (C) Targeted inhibition of mTORC1/2 signaling by PP242 in SRA01/04 cells exhibited a concentration-dependent effect. SRA01/04 cells were incubated with $500 \mathrm{nM}$ Rapa or the indicated doses of PP2 42 for $6 \mathrm{~h}$. Cell lysates were then subjected to western blot analysis to determine the levels of p-AKT (Ser473), AKT, p-mTOR, mTOR, p-p70S6K (Thr389 and Ser371), p70S6K and p-4EBP1 and (D) quantitative analyses of the protein levels was performed. (E) Immunofluorescence analysis of p-AKT (Ser473) (green) in SRA01/04 cells. Representative images indicate that higher PP242 concentrations resulted in lower fluorescence intensities. (scale bar $=100 \mu \mathrm{m}$ ) ${ }^{*} \mathrm{P}<0.05,{ }^{* *} \mathrm{P}<0.01$ vs. control. Con, control; ns, not significant; 4EBP1, 4E-binding protein 1; mTORC1/2, mammalian target of rapamycin complex 1/2; p, phosphorylated; P70S6K, ribosomal protein s6 kinase; Rapa, rapamycin.

of p53; p53 also regulated ectopic apoptosis in the lens of $\mathrm{Rb}$-deficient mice $(21,22)$. To further validate the signaling pathways involved in the inhibition of LEC growth by PP242, the expression levels of p53 in the LECs were analyzed. As presented in Fig. 4A and B, a significant and time-dependent upregulation of p53 in the LECs was detected following treatment with PP242. These results further confirmed that PP242 not only inhibits cell cycle progression by downregulating cyclin D1, but also upregulates p53 in LECs. An increasing number of studies have demonstrated that Bax possesses pro-apoptotic activities, which are activated by $\mathrm{p} 53$, and associates with $\mathrm{Bcl}$ extra large protein in order to open mitochondrial membrane pores, leading to the release of cytochrome $c$ into the cytoplasm (23). Cytochrome $c$ then activates the caspase cascade via apoptotic protease activating factor 1 and caspase-3 (24). Conversely, Bcl-2, which evolved as an important regulator of mitochondrial integrity, is classified as an anti-apoptotic protein (25). As expected, the results of the present study revealed that a gradual downregulation of the anti-apoptotic Bcl-2 occurred with PP242 treatment, leading to an increase in the pro-apoptotic activity of Bax. This result suggested that PP242 may mediate apoptotic signaling via the $\mathrm{Bax} / \mathrm{Bcl}-2$ pathway and that its effect is also associated with increased levels of $\mathrm{p} 53$.

The specific mTORC1/2 inhibitor PP242 causes apoptosis and induces autophagy in LECs. Following treatment with different concentrations of PP242 $(0,0.5,1,1.5$ and $2 \mu \mathrm{M})$ 


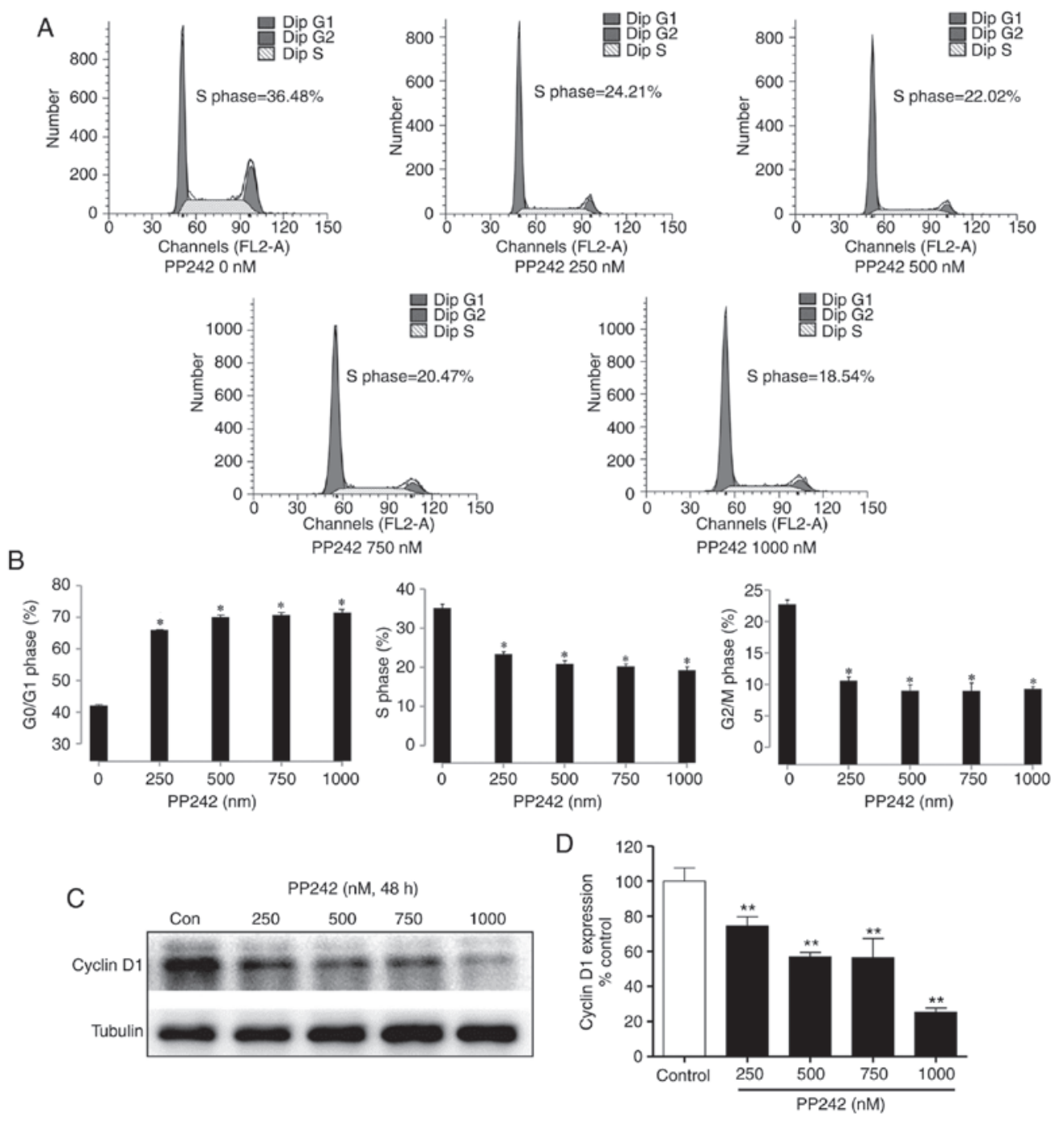

Figure 3. PP242 induces cell cycle arrest at the G0/G1 phase in lens epithelial cells by downregulating cyclin D1. (A and B) SRA01/04 cells were incubated with different concentrations of PP242 (0,250,500,750 and 1,000 nm) for $48 \mathrm{~h}$. Cells were then stained with propidium iodide and analyzed by flow cytometry. The results demonstrated that PP242 increased the number of cells in the G0/G1 phase and reduced the number in S and G2/M phase compared with in the negative control group. (C) Western blot analysis of cyclin D1 and (D) quantitative analysis revealed a marked downregulation in the presence of PP242. ${ }^{*} \mathrm{P}<0.05,{ }^{* *} \mathrm{P}<0.01$ vs. control. Con, control.

for $48 \mathrm{~h}$, the induction of apoptosis was verified using FACS analysis. The percentages of early- and late-stage apoptotic cells are presented in the lower right and upper right quadrants (Q4 and 2, respectively) of the histograms (Fig. 4C). As presented in Fig. 4C, only 5.2\% of the total cells in the control group were apoptotic, whereas in the PP242-treated groups, the percentage of apoptotic cells gradually increased with increasing concentrations of PP242. The total percentage of apoptotic cells $(\mathrm{Q} 2+\mathrm{Q} 4)$ increased from $5.2 \%$ in the PP242-untreated LEC cells to 11.2, 15.1, 21.3 and $28.7 \%$ in the cells treated with $0,0.5,1,1.5$, and $2 \mu \mathrm{M} \mathrm{PP} 242$, respectively, for $48 \mathrm{~h}$. Western blot analysis was then used to analyze signaling induction of LEC apoptosis by PP242. Treatment with PP242 resulted in a marked increase in cleaved caspase-3 and cleaved PARP. Existing evidence has reported that a number of mTOR inhibitors activate autophagy in a variety of cancer cell types (26). To determine whether the mTORC1/2 inhibitor PP242 induced autophagy in LECs, the present study analyzed LC3-I, which is an abundant cytoplasmic protein that is cleaved and degraded, thereby forming LC3-II during the initiation of autophagy. The results of Fig. 4D and E indicated an increase in the ratio of LC3-II/LC3-I, further confirming an increase in autophagy upon mTOR inhibition. Therefore, the results of the present study suggested that PP242 induced LEC autophagy and potentiated cell death.

\section{Discussion}

Understanding how PCO progresses from initiation to end-stage fibrosis by investigating the principal factors and mechanisms that promote the expansion of PCO is critical for establishing novel therapies (27). The defining characteristic of PCO progression is fibrosis, which includes hyperproliferation, migration, matrix deposition, matrix contraction and transdifferentiation of fibroblasts into myofibroblasts. The activation of the AKT/mTOR signaling pathway serves a major role in the initiation of PCO $(28,29)$. Previous studies have demonstrated that numerous growth factors, including transforming growth factor- $\beta$, HGF, IGF and FGF (13-16,30), activate the AKT/mTOR signaling pathway 
A
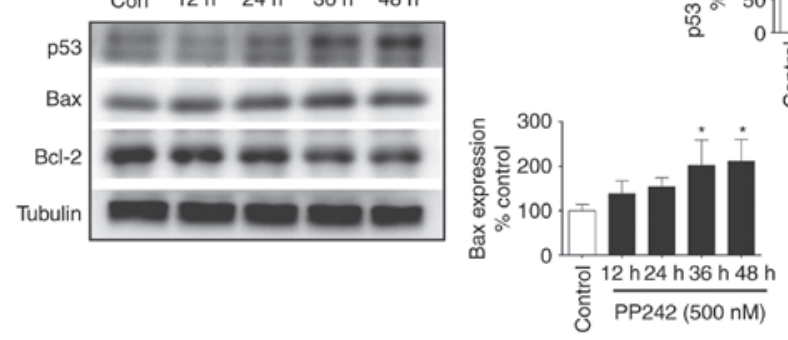

B
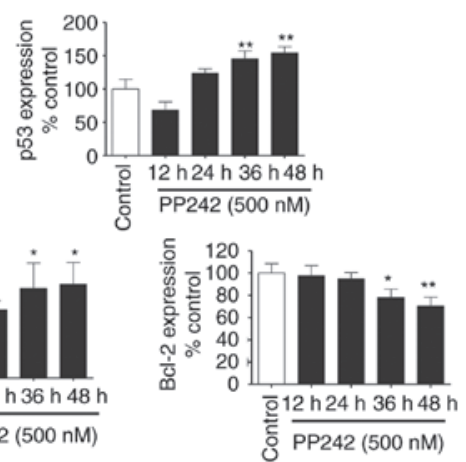

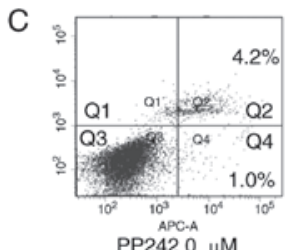

$\mathrm{PP} 2420 \mu \mathrm{M}$



PP242 $0.5 \mu \mathrm{M}$

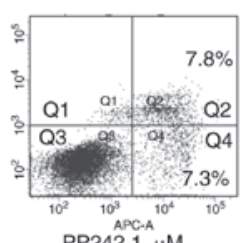

$\mathrm{PP} 2421 \mu \mathrm{M}$
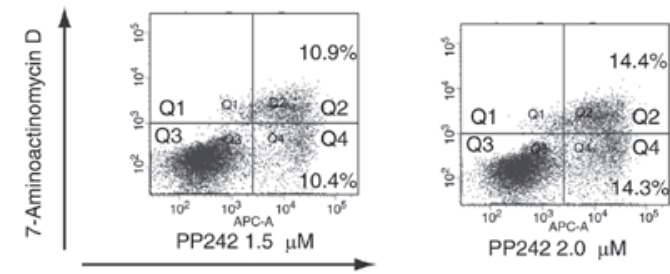

PP242 $2.0 \mu \mathrm{M}$

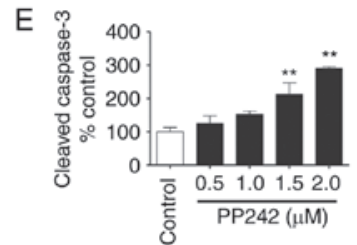

。
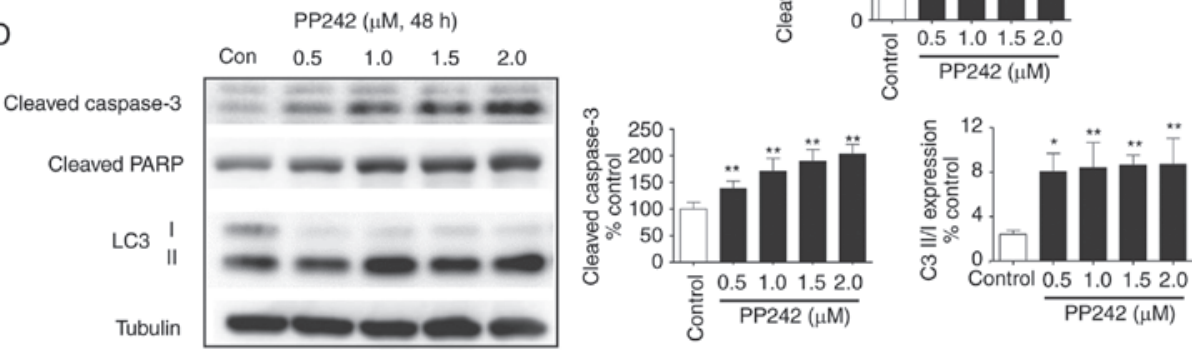

Figure 4. Increased caspase-3-dependent apoptosis upon mTOR inhibition by PP242 treatment in LECs. (A) Effect of PP242 on p53, Bax and Bcl-2 protein expression levels in LECs. SRA01/04 cells were incubated with $500 \mathrm{nM}$ PP242 for 12, 24, 36 and 48 h. Cell lysates were then subjected to western blotting to determine the levels of p53, Bax and Bcl-2. (B) The corresponding quantitative analysis indicated that the levels of p53 and Bax were significantly increased by PP242 in a time-dependent manner, while Bcl-2 gradually decreased. (C) SRA01/04 cells were treated with PP242 (0, 0.5 , 1, 1.5 and $2 \mu \mathrm{M})$ for 48 h in serum-free medium. Annexin-V-APC and 7-Aminoactinomycin D staining were used to quantify the percentage of apoptotic cells. (D) Western blot analysis and (E) the corresponding quantitative analyses revealed that the apoptosis-associated proteins cleaved caspase-3 and cleaved PARP exhibited significant increases induced by PP242 in a dose-dependent manner. The increased LC3 II/I ratio revealed that PP242 also induced autophagy via the inhibition of mTOR. ${ }^{*} \mathrm{P}<0.05,{ }^{* *} \mathrm{P}<0.01$ vs. control. Con, control; Bcl-2, B-cell lymphoma 2; Bax, Bcl-2-associated X; LC3, microtubule-associated protein light chain 3; LECs, lens epithelial cells; mTOR, mammalian target of rapamycin; PARP, poly(ADP ribose) polymerase; APC, allophycocyanin; Q, quadrant.

to induce epithelial-to-mesenchymal transformation, which is a key process that may be triggered by an inflammatory response (31), e.g. the response in the eye following cataract surgery (2).

In cells, mTOR regulates numerous basic characteristics of cell growth and division and is the catalytic subunit of two functionally distinct complexes, namely mTORC1 and mTORC2. mTORC1 is a primary regulator of protein translation phosphorylates S6 kinase and the inhibitory 4EBPs. mTORC2 phosphorylates and regulates AKT, a downstream effector of the PI3K signaling pathway, which mediates growth and survival signals. Collectively, these complexes coordinate a variety of processes that include protein translation, autophagy, proliferation, survival and metabolism in response to nutrient, energy and growth factor signals (32). Rapamycin was originally identified in cancer research as an antifungal compound that targeted $\mathrm{mTORC} 1$ and greatly facilitated the study of mTOR signaling $(33,34)$. In contrast to the in vitro results of the present study, the clinical success of rapamycin has been limited to a few rare cancers, including mantle cell lymphoma, renal cell carcinoma and endometrial cancer (35). Regarding the prevention of PCO, rapamycin was observed to inhibit the proliferation, migration and fibronectin secretion of LECs in vitro and in vivo (36-38); however, no long-term damage to the corneal endothelium due to rapamycin has been reported. In addition, rapamycin was less effective than PP242 in the inhibition of proliferation and migration, and failed to inhibit the phosphorylation of 4EBP1 in SRA01/04 cells in the present study. This indicated that the effects of rapamycin in these LECs were limited. In addition, this may also be the case in clinical trials comparing cancer therapies. Compared with rapamycin, PP242 inhibited mTOR activation 
within SRA01/04 cells, while the phosphorylation of mTOR failed to decrease significantly; however, the expression of phosphorylated AKT S473 increased, demonstrating that the AKT feedback loop was activated.

These limitations, including the incomplete inhibition of mTORC1, the ineffectiveness toward mTORC 2 and the AKT feedback loop as reported in the present study, led to the development of mTORC1/2 dual inhibitors, also known as second-generation mTOR inhibitors (39). PP242 is an example of an active-site inhibitor, which as identified by Feldman et al (40), and which may be used to investigate the selectivity of numerous inhibitors of PI3K scaffold activity (32). In contrast to rapamycin, which targets only certain functions of mTORC1, PP242 inhibits mTORC1 as well as mTORC2. Furthermore, PP242 also inhibits PI3K in addition to inhibiting mTORC1 and mTORC2 (40). In the present study, PP242 effectively reduced LEC proliferation and migration in a dose-dependent manner. The phosphorylation of AKT S473 was markedly inhibited by PP242, which demonstrated that PP242 may inhibit mTORC2 in the LECs. The significant downregulation of p-p70S6K (Thr389 and Ser371) and p-4EBP1 indicated that mTORC1 was almost completely blocked by PP242 in the LECs even at low concentrations and for a short duration. The present study reported that the action of PP242 in LECs was more effective than that of rapamycin, similar to the results of previous studies on other cell types $(39,40)$.

Furthermore, in addition to studying the inhibition of proliferation and migration by PP242, alterations in the cell cycle of PP242-treated LECs were assessed by flow cytometry. The present study revealed that PP242 induced the cell cycle in LECs by downregulating cyclin D1. In normal cells, p53 is known as a tumor suppressor gene that controls responses to numerous different cellular stresses, including DNA damage, hypoxia and oncogene activation (41). In the present study, the levels of p53 markedly increased in a time-dependent manner following PP242 treatment, suggesting that PP242 may inhibit cell growth by regulating p53. In addition, the gradual downregulation of anti-apoptotic $\mathrm{Bcl}-2$ was also observed in response to PP242 treatment, leading to an increase in the pro-apoptotic activity of Bax. The marked increase in Bax expression following PP242 treatment of the LEC, along with the reduction of Bcl-2 expression, confirmed that following treatment of the LECs with PP242, the cells ceased to proliferate and underwent apoptosis. The cells were unprotected from the apoptotic stimuli of Bax by the attenuation of expression of the $\mathrm{Bcl}-2$ protein. Accumulating evidence revealed that abnormal growth or apoptotic cell death in the lens epithelium significantly alters cataract formation $(42,43)$. Hence, the apoptosis of HLECs is one of the most crucial pathologic factors of cataracts. On the contrary, the apoptosis or cell death of residual HLECs following cataract surgery is an effective strategy in preventing PCO. The intraocular HLECs at the equator and under the anterior lens capsule may be removed as much as possible in cataract surgery. Despite advanced technologies and equipment in cataract surgical procedures and IOL design, it is difficult to remove HLECs. This situation promotes the use of post-operative pharmacotherapy for inhibiting the proliferation of HLECs and promoting apoptosis or cell death. Post-operative pharmacotherapy is expected to become an important means of treatment of PCO. In the present study, FACS analysis demonstrated an increased caspase-3-dependent apoptosis upon the inhibition of mTOR by PP242 treatment of LECs. The results of present study indicated that the dual mTORC1/2 inhibitor PP242 suppressed LEC proliferation and migration. Collectively, due to its ability to induce LEC apoptosis, PP242 may be considered as a candidate drug for the prevention of PCO.

As mTOR controls numerous cellular processes, including autophagy (44), this process was also taken into consideration in the present study. Autophagy protects cells, but this process may also mediate cell death or develop as a primary response to stress inducing cell death, depending on the specific circumstances $(45,46)$. mTOR promotes cell death and results in deterioration in certain diseases. In mammalian cells, autophagy genes or the autophagy signaling pathway are necessary for a non-apoptotic cell death pathway; it is possible that the autophagy pathway may act as a tumor suppressor by causing autophagic cell death (47). It was observed in the present study that PP242 induced and increased the conversion of LC3-I to LC3-II, accompanied by increased levels of cleaved-caspase-3 and cleaved-PARP in the LECs, indicating that autophagy is involved in PP242-mediated LEC death. Further study is required to determine the complex regulatory role of autophagy and mTOR signaling in the death of LECs.

The present study revealed that PP242 markedly inhibited mTORC1 and mTORC2-mediated signaling activation, suppressed the proliferation and migration of LECs, and induced cell apoptosis in vitro. Further in vivo experimental study of $\mathrm{PCO}$ is required to support the potential clinical application of PP242. PP242 has been tested in cancer therapy in vivo. Atreya et al (48) reported that $5.8 \mu \mathrm{M}$ PP242 may be detected in the plasma following an oral dose of $2.5 \mathrm{mg}$ PP2 42 in mice. This indicated that compared with the $\mathrm{IC}_{50}(2.159 \mu \mathrm{M})$ of PP242 in the SRA01/04 cell line tested in the present study in vitro, the limitation that the $\mathrm{IC}_{50}$ of the inhibitor may exceed pharmacologically relevant concentrations is unlikely to apply to PP242. In addition, partial or temporary TORC1/2 kinase inhibition is tolerated by normal lymphocytes (49). Local therapies, including eye drops or intraocular injection, are prefered for eye disease treatments. Therefore, localized therapy using this route of PP242 administration may maximally limit the risk of PP242 toxicity to other organs. Based on the aforementioned evidence, PP242 may have potential for in vivo application in the future. In conclusion, the active-site inhibitor PP242 requires further investigation as a potential strategy for drug treatment in the prevention of PCO.

\section{Acknowledgements}

The present study was supported by the Liaoning Province Science and Technology Plan Project (grant no. 2013225303) and the Liaoning Provincial Natural Science Foundation (grant no. 2013021029).

\section{Competing interests}

The authors declare that they have no competing interests.

\section{References}

1. Apple DJ, Solomon KD, Tetz MR, Assia EI, Holland EY, Legler UF, Tsai JC, Castaneda VE, Hoggatt JP and Kostick AM: Posterior capsule opacification. Surv Ophthalmol 37: 73-116, 1992. 
2. Wormstone IM, Wang L and Liu CS: Posterior capsule opacification. Exp Eye Res 88: 257-269, 2009.

3. Awasthi N, Guo S and Wagner BJ: Posterior capsular opacification: A problem reduced but not yet eradicated. Arch Ophthalmol 127: 555-562, 2009.

4. Chan E, Mahroo OA and Spalton DJ: Complications of cataract surgery. Clin Exp Optom 93: 379-389, 2010.

5. Nishi O: Influence of intraocular lens material and design on the development of posterior capsule opacification. Ophthalmologe 102: 572-578, 2005 (In German).

6. Nishi O, Yamamoto N, Nishi K and Nishi Y: Contact inhibition of migrating lens epithelial cells at the capsular bend created by a sharp-edged intraocular lens after cataract surgery. J Cataract Refract Surg 33: 1065-1070, 2007.

7. Apple DJ, Peng Q, Visessook N, Werner L, Pandey SK, Escobar-Gomez M, Ram J and Auffarth GU: Eradication of posterior capsule opacification: Documentation of a marked decrease in Nd:YAG laser posterior capsulotomy rates noted in an analysis of 5416 pseudophakic human eyes obtained postmortem. Ophthalmology 108: 505-518, 2001

8. Javitt JC, Tielsch JM, Canner JK, Kolb MM, Sommer A and Steinberg EP: National outcomes of cataract extraction. Increased risk of retinal complications associated with Nd:YAG laser capsulotomy. The cataract patient outcomes research team. Ophthalmology 99: 1487-1498, 1992.

9. Nibourg LM, Gelens E, Kuijer R, Hooymans JM, van Kooten TG and Koopmans SA: Prevention of posterior capsular opacification. Exp Eye Res 136: 100-115, 2015.

10. Zoncu R, Efeyan A and Sabatini DM: mTOR: From growth signal integration to cancer, diabetes and ageing. Nat Rev Mol Cell Biol 12: 21-35, 2011.

11. Guertin DA and Sabatini DM: An expanding role for mTOR in cancer. Trends Mol Med 11: 353-361, 2005.

12. Cargnello M, Tcherkezian J and Roux PP: The expanding role of mTOR in cancer cell growth and proliferation. Mutagenesis 30 : 169-176, 2015

13. Tian F, Dong L, Zhou Y, Shao Y, Li W, Zhang H and Wang F: Rapamycin-induced apoptosis in HGF-stimulated lens epithelial cells by AKT/mTOR, ERK and JAK2/STAT3 pathways. Int J Mol Sci 15: 13833-13848, 2014.

14. Xiong W, Cheng BH, Jia SB and Tang LS: Involvement of the PI3K/Akt signaling pathway in platelet-derived growth factor-induced migration of human lens epithelial cells. Curr Eye Res 35: 389-401, 2010.

15. Wederell ED and de Iongh RU: Extracellular matrix and integrin signaling in lens development and cataract. Semin Cell Dev Biol 17: 759-776, 2006.

16. Teo ZL, McQueen-Miscamble L, Turner K, Martinez G, Madakashira B, Dedhar S, Robinson ML and de Iongh RU: Integrin linked kinase (ILK) is required for lens epithelial cell survival, proliferation and differentiation. Exp Eye Res 121: 130-142, 2014.

17. Sparks CA and Guertin DA: Targeting mTOR: Prospects for mTOR complex 2 inhibitors in cancer therapy. Oncogene 29: 3733-3744, 2010.

18. Schenone S, Brullo C, Musumeci F, Radi M and Botta M ATP-competitive inhibitors of mTOR: An update. Curr Med Chem 18: 2995-3014, 2011

19. Weber JD and Gutmann DH: Deconvoluting mTOR biology. Cell Cycle 11: 236-248, 2012

20. Vousden KH and Prives C: Blinded by the Light: The growing complexity of p53. Cell 137: 413-431, 2009.

21. Chen Q, Ash JD, Branton P, Fromm L and Overbeek PA: Inhibition of crystallin expression and induction of apoptosis by lens-specific E1A expression in transgenic mice. Oncogene 21 : 1028-1037, 2002

22. Morgenbesser SD, Williams BO, Jacks T and DePinho RA: p53-dependent apoptosis produced by $\mathrm{Rb}$-deficiency in the developing mouse lens. Nature 371: 72-74, 1994

23. Malecaze F, Decha A, Serre B, Penary M, Duboue M, Berg D, Levade T, Lubsen NH, Kremer EJ and Couderc B: Prevention of posterior capsule opacification by the induction of therapeutic apoptosis of residual lens cells. Gene Ther 13: 440-448, 2006.

24. Pataer A, Fang B, Yu R, Kagawa S, Hunt KK, McDonnell TJ, Roth JA and Swisher SG: Adenoviral Bak overexpression mediates caspase-dependent tumor killing. Cancer Res 60: 788-792, 2000

25. Zheng JH, Viacava Follis A, Kriwacki RW and Moldoveanu T: Discoveries and controversies in BCL-2 protein-mediated apoptosis. FEBS J 283: 2690-2700, 2016.
26. Munson MJ and Ganley IG: MTOR, PIK3C3, and autophagy: Signaling the beginning from the end. Autophagy 11: 2375-2376, 2015.

27. Wormstone IM and Eldred JA: Experimental models for posterior capsule opacification research. Exp Eye Res 142: 2-12, 2016.

28. Liegl R, Wertheimer C, Kernt M, Docheva D, Kampik A and Eibl-Lindner KH: Attenuation of human lens epithelial cell spreading, migration and contraction via downregulation of the PI3K/Akt pathway. Graefes Arch Clin Exp Ophthalmol 252: 285-292, 2014.

29. Laplante M and Sabatini DM: mTOR signaling at a glance. J Cell Sci 122: 3589-3594, 2009.

30. Guo R, Meng Q, GuoH, Xiao L, Yang X, Cui Y andHuang Y: TGF- $\beta 2$ induces epithelial-mesenchymal transition in cultured human lens epithelial cells through activation of the PI3K/Akt/mTOR signaling pathway. Mol Med Rep 13: 1105-1110, 2016.

31. López-Novoa JM and Nieto MA: Inflammation and EMT: An alliance towards organ fibrosis and cancer progression. EMBO Mol Med 1: 303-314, 2009

32. Liu Q, Thoreen C, Wang J, Sabatini D and Gray NS: mTOR mediated anti-cancer drug discovery. Drug Discov Today Ther Strateg 6: 47-55, 2009.

33. Sehgal SN, Baker H and Vézina C: Rapamycin (AY-22,989), a new antifungal antibiotic. II. Fermentation, isolation and characterization. J Antibiot (Tokyo) 28: 727-732, 1975.

34. Rao RD, Buckner JC and Sarkaria JN: Mammalian target of rapamycin (mTOR) inhibitors as anti-cancer agents. Curr Cancer Drug Targets 4: 621-635, 2004.

35. Houghton PJ: Everolimus. Clin Cancer Res 16: 1368-1372, 2010.

36. Liu H, Feng G, Wu L, Fu S, Liu P, Yang W and Zhang X: The effects of rapamycin on lens epithelial cell proliferation, migration, and matrix formation: An in vitro study. Mol Vis 16: 1646-1653, 2010

37. Liu H, Wu L, Fu S, Hou Y, Liu P, Cui H, Liu J, Xing L and Zhang X: Polylactide-glycoli acid and rapamycin coating intraocular lens prevent posterior capsular opacification in rabbit eyes. Graefes Arch Clin Exp Ophthalmol 247: 801-807, 2009.

38. Wang $\mathrm{Z}$ and Wang Z: Effects of rapamycin on expression of Bcl-2 and Bax in human lens epithelial cells and cell cycle in rats. J Huazhong Univ Sci Technolog Med Sci 31: 555-559, 2011

39. Zhou HY and Huang SL: Current development of the second generation of mTOR inhibitors as anticancer agents. Chin J Cancer 31: 8-18, 2012

40. Feldman ME, Apsel B, Uotila A, Loewith R, Knight ZA, Ruggero D and Shokat KM: Active-site inhibitors of mTOR target rapamycin-resistant outputs of mTORC1 and mTORC2. PLOS Biol 7: e38, 2009.

41. Muller PA and Vousden KH: Mutant p53 in cancer: New functions and therapeutic opportunities. Cancer Cell 25: 304-317, 2014.

42. Osnes-Ringen $\varnothing$, Berg KH, Moe MC, Zetterström C, Røger M and Nicolaissen B: Cell death pattern in lens epithelium of cataract patients. Acta Ophthalmol 94: 514-520, 2016.

43. Li Y, Liu S, Zhang F, Jiang P, Wu X and Liang Y: Expression of the microRNAs hsa-miR-15a and hsa-miR-16-1 in lens epithelial cells of patients with age-related cataract. Int J Clin Exp Med 8: 2405-2410, 2015

44. Lin Z, McDermott A, Shao L, Kannan A, Morgan M, Stack BC Jr, Moreno M, Davis DA, Cornelius LA and Gao L: Chronic mTOR activation promotes cell survival in Merkel cell carcinoma. Cancer Lett 344: 272-281, 2014.

45. Baehrecke EH: Autophagic programmed cell death in Drosophila. Cell Death Differ 10: 940-945, 2003.

46. Tresse E, Kosta A, Luciani MF and Golstein P: From autophagic to necrotic cell death in Dictyostelium. Semin Cancer Biol 17: 94-100, 2007

47. Yu L, Alva A, Su H, Dutt P, Freundt E, Welsh S, Baehrecke EH and Lenardo MJ: Regulation of an ATG7-beclin 1 program of autophagic cell death by caspase-8. Science 304: 1500-1502, 2004

48. Atreya CE, Ducker GS, Feldman ME, Bergsland EK, Warren RS and Shokat KM: Combination of ATP-competitive mammalian target of rapamycin inhibitors with standard chemotherapy for colorectal cancer. Invest New Drugs 30: 2219-2225, 2012.

49. Janes MR, Limon JJ, So L, Chen J, Lim RJ, Chavez MA, Vu C, Lilly MB, Mallya S, Ong ST, et al: Effective and selective targeting of leukemia cells using a TORC1/2 kinase inhibitor. Nat Med 16: 205-213, 2010

This work is licensed under a Creative Commons Attribution-NonCommercial-NoDerivatives 4.0 International (CC BY-NC-ND 4.0) License. 